\section{JOINT INSTITUTE FOR AERONAUTICS AND ACOUSTICS}

NCC2-173 +NCC2--

\author{
JIAA TR - 70
}

National Aeronautics and
Space Administration

Ames Research Center

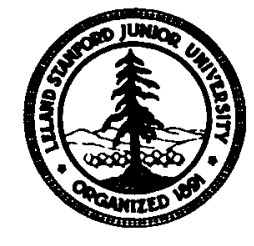

Stanford University

\title{
A THEORY FOR TURBULENT CURVED WALL JETS
}

BY

Leonard Roberts

\author{
Stanford University \\ Department of Aeronautics and Astronautics \\ Stanford, CA 94305
}

\section{MARCH 1986}

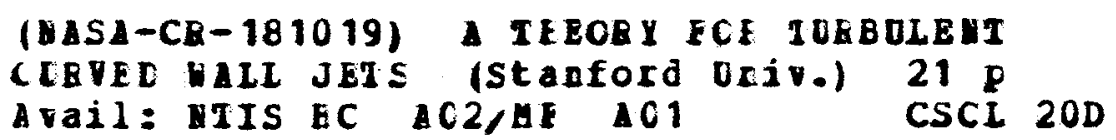

$187-22951$

Unclas

$63 / 340077693$ 
JIAA TR - 70

\section{A THEORY FOR TURBULENT CURVED WALL JETS}

BY

Leonard Roberts

Stanford University

Department of Aeronautics and Astronautics Stanford, CA 94305 


\begin{abstract}
A simple theoreticai model is proposed to describe the flow of a turbulent wall jet along a curved surface into a quisecent atmosphere. An integral method is used to solve the momentum equation and identifies three contributions to the spreading rate of the wall jet: (1) turbulent diffusion in the wall jet, (2) wall curvature and (3) rate of change of wall curvature. Closed from approximate solutions are found for the case of a plane wall, a circular cylinder and a logarithmic spiral surface and comparison with experimental data for these three cases is made showing good agreement between theory and experiment.
\end{abstract}




\section{ACKNOWLEDGEMENTS}

This work was supported by NASA Contracts NCC 2-173 and NCC 2-55. 


\section{Introduction}

The behavior of a wall jet flowing around a curved surface has been the subject of study for almost two hundred years following the first observations of Young(1) in 1800 . Practical application of this phenomena, popularly known as the Coanda effect, have been pursued in more recent times with a view to delaying separation of the boundary layer on lifting surfaces by using a wall jet over a rounded trailing edge: the wall jet causes the flow to remain attached to the surface, displaces the rear stagnation point and induces additional circulation, and therefore produces additional lift on the surface. The use of this phenomena in the design of circulation control wings (CCW) has received considerable attention in recent years and has been described by a number of investigators (see for example Wood ${ }^{(2)}$ and Englar $\left.{ }^{(3)}\right)$.

Despite the fact that the phenomena is being used in practical applications to wing and rotor design the understanding of the properties of wall jets is still limited and depends primarily in experimental information regarding the turbulent mixing, its effect on the spreading rate of the wail jet and the corresponding deceleration of the flow as it proceeds along the wall. A thorough review was made by Launder and Rodi ${ }^{(4)}$ in 1981 of available data for wall jets adjacent to surfaces of plane, cylinder and logarithmic spiral surfaces and substantial use may be made of this information to distinguish the influence of wall curvature, and rate of change of curvature in the stream direction, on the spreading rate of the jet. This information together with data from the same review on the mean velocity profile in the wall jet provides the basis for checking theoretical models of the flow.

In the present paper an attempt is made to formulate the simplest possibie model for the flow of a wall jet emanating from a two dimensional source into quiescent surroundings in the presence of a wall of arbitrary shape. The method uses self similar profiles for the mean velocity together with a simple eddy viscosity model. The streamwise and radial momentum equations are integrated across the wall jet flow to give an expression for the 
momentum balance including the effect of the pressure gradient induced by the rate of change of surface curvature. The streamwise momentum equation is also evaluated at the point of maximum velocity to provide a second equation and thereby permit a solution for the two unknown quantities $b$, the jet half width and $u_{m}$, the maximum velocity. This approach provides approximate closed form solutions for the flow of the wall jet over surfaces of various shapes (for quiescent surroundings), and in particular permits a direct comparison with the available experimental results for plane, cylindrical or logarithmic spiral surfaces.

\section{Analysis}

The wall jet is considered to comprise two parts: an inner flow adjacent to the wall having a highly non-linear velocity profile characteristic of a turbulent wall flow, and an outer flow having a velocity profile more typical of a free turbulent plane jet.

The primary parameters that describe the flow are shown in figure 1 . The jet emerges from a point source into a fluid at rest and spreads, increasing its width and decreasing its velocity due to turbulent diffusion in the jet and friction at the wall. At a distance $s$ downstream of the jet exit the velocity to can be expressed as

$$
u=u_{m}(s) f(y / b)
$$

where $u_{m}$ is the maximum velocity, occurring at $y=y_{m}(s)$, and $b=b(s)$ is the half width of the jet (at which point $u=\frac{1}{2} u_{m}$ ).

The velocity profile in the outer flow $\left(y>y_{m}\right)$ is assumed to take the form:

$$
u=u_{m} \operatorname{sech}^{2}\left[\frac{k\left(\xi-\xi_{m}\right)}{1-\xi_{m}}\right], \xi>\xi_{m}
$$


where $\xi=y / b$. This velocity profile is suggested by the classical free jet solution found by Tollmien, modified to give $u=u_{m}$ at $\xi=\xi_{m}$. The constant $k$ is determined such that $u=\frac{1}{2} u_{m}$ at $\xi=1(y=b)$, thus

$$
k=\tanh ^{-1}\left(\frac{1}{\sqrt{2}}\right)=.8814
$$

The velocity profile for the inner flow is assumed to depend on the variable $\left(\xi / \xi_{m}\right)^{\frac{1}{n}}$ as suggested by turbulent wall flow, and is chosen to give a maximum value $u=u_{m}$ at $\xi=\xi_{m}:$ the following profile satisfies these conditions

$$
u=u_{m}\left[2\left(\frac{\xi}{\xi_{m}}\right)^{\frac{1}{n}}-\left(\frac{\xi}{\xi_{m}}\right)^{\frac{2}{n}}\right]
$$

The value $\xi_{m}$, giving the location of maximum velocity, is determined by matching the second derivative of the velocity profiles as given by equations (2) and (4), (the first derivative is zero since this is the maximum velocity point).

The result is written

$$
\xi_{m}=(1+k n)^{-1}
$$

Before proceeding with the analysis, a comparison is made in figure 2 of the velocity profiles with the experimental data of Tailland ${ }^{(5)(8)}$. The data are typical of those taken by a number of authors for wall jet flows over plane and curved surfaces and show that the assumed profile given by equations (2) and (4) is reasonable. Additionally in figure 3 a comparison is made of $\xi_{m}$, given by equation (5), with the experimentally determined values given by Forthmann ${ }^{(7)}$, Sigalla $^{(8)}$ Bradshaw \& Gill ${ }^{(9)}$, Patel $^{(10)}$ and Giles ${ }^{(1)}$. All the data falls within a band $.14<\xi_{m}<.16$ corresponding to $7>n>6$ for Reynolds number 
$R_{0}$ in the range $10^{4}$ to $10^{5} ;$ thus the values of $n$ are consistent with those expected for turbulent wall flows.

In this paper the two primary flow variables to be determined are the jet half width, b, and the maximum velocity $u_{m}$. The continuity and momentum equations for an incompressible fluid are written

$$
\begin{gathered}
\frac{\partial u}{\partial s}+\frac{\partial}{\partial y}(h v)=0 \\
h u \frac{\partial u}{\partial s}+h v \frac{\partial}{\partial y}(h u)=\frac{-h}{\rho} \frac{\partial p}{\partial s}+\frac{\partial}{\partial y}\left(h^{2} \tau\right)
\end{gathered}
$$

and

$$
\frac{u^{2}}{R}=\frac{1}{\rho} h \frac{\partial p}{\partial y}
$$

where

$$
h=1+y / R
$$

Equations (6) to (8) can be combined to give

$$
\frac{\partial}{\partial s}\left(u^{2}\right)+\frac{u^{2} y}{R^{2}} \frac{d R}{d s}=\frac{\partial}{\partial y}\left(h^{2} \tau-h^{2} u v-\frac{\partial}{\partial s}\left(\frac{p y}{\rho}\right)\right)
$$

neglecting terms of $0\left(\frac{1}{R^{2}}\right)$. Integration across the flow gives the integral form of the momentum equation:

$$
\frac{d}{d s}\left[\int_{0}^{\infty} u^{2} d y\right]+\frac{1}{R^{2}} \frac{d R}{d s}\left[\int_{o}^{\infty} u^{2} y d y\right]=-\tau_{o} / \rho
$$


This equation retains the term which reflects the curvature-induced pressure gradient which may be large compared with $\tau_{0} / \rho$, the wall shear stress.

Substitution of the velocity profiles (equations 2 and 4 ) into the integrals of equation (10) gives

$$
A_{n}\left[\frac{1}{u_{m}^{2}} \frac{d}{d s}\left(b u_{m}^{2}\right)\right]+C_{n}\left[\frac{b^{2}}{R^{2}} \frac{d R}{d s}\right]=-\frac{1}{2} C_{f}
$$

where

$$
\begin{aligned}
& A_{n}=\left(1+\frac{2}{3} n\right)(1+k n)^{-1} \\
& C_{n}=\frac{1}{2}\left[\left(\frac{4}{3} \log 2-\frac{1}{3}\right) n^{2}+\frac{4}{3} n+1\right](1+k n)^{-2}
\end{aligned}
$$

From equation (11) it can be seen that in the absence of curvature effects the wall jet momentum $\left(b u_{m}^{2}\right)$ decreases with distance due to skin friction at the wall. However for a wall of decreasing radius of curvature $\left(\frac{d R}{d \iota}<0\right)$ it is possible for the wall jet momentum to increase with distance along the wall. This will occur if

$$
C_{n} \frac{b^{2}}{R^{2}}\left(-\frac{d R}{d s}\right)>\frac{1}{2} C_{f}
$$

An approximate form of equation (11) valid for large $n$ and for $C_{f} \rightarrow 0$ may be written

$$
\frac{1}{u_{m}^{2}} \frac{d}{d s}\left(b u_{m}^{2}\right)+\frac{\left(\log 2-\frac{1}{4}\right)}{k} \frac{b^{2}}{R^{2}} \frac{d R}{d S}=0
$$

a result which is applicable for large Reynolds number.

Since there are two unknown quantities in equation (11) it is necessary to use a second relationship in order to determine $b$ and $u_{m}$. It is convenient to use the momentum equation 
(7) evaluated at the point of maximum velocity, (ie where $u=u_{m}, \frac{\partial u}{\partial y}=0$ ) which may be written

$$
\left[u \frac{\partial u}{\partial s}+\frac{u v}{R}+\frac{1}{\rho} \frac{\partial p}{\partial s}\right]_{m}=\left[\frac{h}{\rho} \frac{\partial \tau}{\partial y}+\frac{2 \tau}{\rho R}\right]_{m}
$$

The terms on the left hand side may be written in dimensionless form, neglecting terms of $O(b / R)^{2}$ as follows:

$$
\frac{b}{u_{m}^{2}}\left(u \frac{\partial u}{\partial s}\right)_{m}=\frac{1}{2}\left[\frac{1}{u_{m}^{2}} \frac{d}{d s}\left(b u_{m}^{2}\right)-\frac{d b}{d s}\right]
$$

and

$$
\begin{aligned}
\frac{b}{u_{m}^{2}}\left(\frac{u v}{R}\right) & =\frac{1}{u_{m}}\left[-\int_{0}^{y m} \frac{\partial u}{\partial s} d y\right] b / R \\
& =-\frac{1}{2(1+k n)}\left[\frac{1}{u_{m}^{2}} \frac{d}{d s}\left(b u_{m}^{2}\right)-\frac{d b}{d s}\right] b / R
\end{aligned}
$$

Finally,

$$
\begin{aligned}
\frac{b}{u_{m}^{2}} \cdot \frac{1}{\rho} \frac{\partial p}{\partial s} & =-\frac{b}{u_{m}^{2}} \frac{d}{d s}\left[\int_{y_{m}}^{\infty} \frac{u^{2}}{R} d y\right] \\
& =-\frac{b}{u_{m}^{2}} \frac{d}{d s}\left(\frac{b u_{m}^{2}}{R} \int_{\xi_{m}}^{\infty}\left(\frac{u}{u_{m}}\right)^{2} d \xi\right) \\
& =-\frac{\frac{2}{3} n}{1+k n} \cdot \frac{b}{R} \cdot\left[\frac{1}{u_{m}^{2}} \frac{d}{d s}\left(b u_{m}^{2}\right)-\frac{b}{R} \frac{d R}{d s}\right]
\end{aligned}
$$

Thus the left hand side of equation (12) becomes

$$
\frac{1}{2}\left(1-\frac{1+\frac{4}{3} n}{1+k n} b / R\right)\left[\frac{1}{u_{m}^{2}} \frac{d}{d s}\left(b u_{m}^{2}\right)\right]-\frac{1}{2}\left(1-\frac{1}{1+k n} b / R\right) \frac{d b}{d s}
$$




$$
+\frac{2}{3}\left(\frac{n}{1+k n}\right) \frac{b^{2}}{R^{2}} \frac{d R}{d s}
$$

In the limiting case of large $n$ this expression simplifies to

$$
\frac{1}{2}\left\{\left(1-\frac{4}{3 k} b / R\right) \frac{1}{u_{m}^{2}} \frac{d}{d s}\left(b u_{m}^{2}\right)-\frac{d b}{d s}+\frac{4}{3 k} b^{2} / R^{2} \frac{d R}{d s}\right\}
$$

In order to evaluate the right hand side of equation (12) it is necessary to express $\tau$ in terms of $u$ through an eddy viscosity $\epsilon$ based on a length scale $b\left(1-\xi_{m}\right)$ and a characteristic velocity $u_{m}$. It is convenient to write

$$
\epsilon=\frac{K}{4 k^{2}} \rho\left(1-\xi_{m}\right) b u_{m} g(\xi)
$$

where $K$ is a constant to be determined experimentally. The function $g(\xi)$ is chosen such that $g\left(\xi_{m}\right)=1$ and also satisfies the appropriate boundary condition at $\xi=0$.

The shear stress takes the form

$$
\frac{\tau}{\rho}=\epsilon\left(\frac{\partial u}{\partial y}-\frac{u}{R}\right)
$$

or, substituting for $u=u_{m} f(\xi)$ and $\epsilon$

$$
\frac{\tau}{\rho u_{m}^{2}}=\frac{K}{4 k^{2}}\left(1-\xi_{m}\right)\left[f^{\prime} g-b / R f g\right]
$$

Similarly,

$$
\frac{b}{\rho u_{m}^{2}} \frac{\partial \tau}{\partial y}=\frac{K}{4 k^{2}}\left(1-\xi_{m}\right)\left[\left(f^{\prime \prime} g+f^{\prime} g^{\prime}\right)-\frac{b}{R}\left(f^{\prime} g+f g^{\prime}\right)\right]
$$

where I denotes a derivative with respect to $\xi$. 
Since $f\left(\xi_{m}\right)=g\left(\xi_{m}\right)=1$ and $f^{\prime}\left(\xi_{m}\right)=O$ the foregoing expressions evaluated at $\xi=\xi_{m}$ combine to give:

$$
\left(\frac{h}{\rho} \frac{\partial \tau}{\partial y}+\frac{2 \tau}{\rho R}\right)_{m}=\frac{u_{m}^{2}}{b}\left\{\frac{K}{4 k^{2}}\left(1-\xi_{m}\right)\left(1+\xi_{m} b / R\right) f_{m}^{\prime \prime}\left(1-b / R g^{\prime} / f^{\prime \prime}\right)_{m}+O(b / R)^{2}\right\}
$$

The quantity $f^{\prime \prime}$ is evaluated from

$$
\begin{array}{r}
f=\operatorname{sech}^{2}\left[\frac{k\left(\xi-\xi_{m}\right)}{1-\xi}\right] \\
f^{\prime \prime}\left(\xi_{m}\right)=-\frac{2 k^{2}}{\left(1-\xi_{m}\right)^{2}}
\end{array}
$$$$
\text { as }
$$

Turning now to the function $g(\xi)$ which describes the variation with $\xi$ of the eddy viscosity, $g(\xi)$ is assumed to take the form:

$$
g(\xi)=\left(\xi / \xi_{m}\right)^{m}\left(a+(1-a) \xi / \xi_{m}\right)
$$

where the exponent $m$ and the constant $a$ are determined by satisfying the condition that

$$
\frac{\tau}{\rho u_{m}^{2}}=\epsilon\left(\frac{\partial u}{\partial y}-\frac{u}{R}\right)=\frac{1}{2} C_{f} \text { at } \xi=0
$$

Substitution for $\epsilon$ and $u$ gives

$$
\frac{K}{4 k^{2}} \frac{1-\xi_{m}}{\xi_{m}} 2 / n a\left(\frac{\xi}{\xi_{m}}\right)^{m+\frac{1}{n}-1}=\frac{1}{2} C_{f}
$$


at $\xi=0$.

Thus

$$
m=1-\frac{1}{n} \text { and } a=\frac{C_{f} k}{K}
$$

(since $\left.\frac{1-\xi_{m}}{\xi_{m}}=k n\right)$

Thus

$$
g(\xi)=\left(\frac{\xi}{\xi_{m}}\right)^{l-\frac{1}{n}}\left[\frac{C_{f} k}{K}+\left(1-\frac{C_{f} k}{K}\right) \frac{\xi}{\xi_{m}}\right]
$$

and $g^{\prime}\left(\xi_{m}\right)$ is evaluated as

$$
g^{\prime}\left(\xi_{m}\right)=\left(2-\frac{1}{n}-\frac{C_{f} k}{K}\right)(1+k n)
$$

Thus equation 17 becomes

$$
\frac{2 b}{u_{m}^{2}}\left(\frac{h}{\rho} \frac{\partial \tau}{\partial y}+\frac{2 \tau}{\rho R}\right)_{m}=-K \cdot \frac{k n}{1+k n}\left\{1+\frac{\left[1-n / 2+n^{2}\left(1-\frac{\sigma_{1} k}{2 K}\right)\right]}{1+k n} \delta / R\right\}
$$

which has the form, for $n$ large and $C_{f} \rightarrow 0$ :

$$
\frac{2 b}{u_{m}^{2}}\left(\frac{h}{\rho} \frac{\partial T}{\partial y}+\frac{2 T}{\rho R}\right)=-K\left[1+\frac{n}{k} b / R\right]
$$

Substitution into equation (12), and elimination of the quantity $\frac{1}{u_{m}^{2}} \frac{d}{d \theta}\left(b u_{m}^{2}\right)$ using equation (11a) gives finally, an expression for the spreading rate $\frac{d b}{d s}$ :

$$
\frac{d b}{d s}=K\left[1+\frac{n}{k} b / R\right]+\left(\frac{19}{12}-\frac{\log 2}{k}\right)\left(b / R \frac{d R}{d s}\right) b / R+O(b / R)^{2}
$$

In this equation $K$ is to be determined experimentally for the flat plate $(\boldsymbol{R} \rightarrow \infty)$ and the term involving $\left(b / R \frac{d R}{d o}\right)$ is retained since this may be the same order as $\frac{d b}{d S}$ (for a log spiral shape, in fact $\left(b / R \frac{d R}{d b}\right) \equiv \frac{d b}{d s}$ 
It is clear from equation 22 that the influence of small curvature (small $b / R$ ) may be quite significant since the quantity $\frac{n}{k} \cdot b / R$ appears in the expression for the spreading rate.

\section{Comparison with Experimental Results}

In order to validate the foregoing anaiysis a comparison is made of the spreading rate as given by equation (22) with available experimental results for two cases, namely, the circular cylinder $(R=$ constant $)$ and the logarithmic spiral $\left(\frac{d R}{d S}=\right.$ const $)$.

\section{(a) Circular Cylinder}

In this case equation 22 reduces to a linear differential equation for $b / R$ namely

$$
\frac{d b}{d s}=K\left[1+\frac{n}{k} b / R\right]
$$

The solution to this equation may be written in the form

$$
\frac{b}{s}=K \frac{\frac{n}{k} \cdot b / R}{\log \left(1+\frac{n}{k} b / R\right)}
$$

or, neglecting terms of $O(b / R)^{2}$,

$$
b / s=K\left(1+\frac{n}{2 k} b / R\right)
$$

The constant $\mathrm{K}$ is determined from the flat plate wall jet $(b / R \equiv O)$ as $\mathrm{K}=.07$, and equation (23a) with $k=.8814$ gives

$$
b / s=07(1+4 b / R) \quad(n=7)
$$




$$
b / s=.07(1+3.4 b / R) \quad(n=6)
$$

These expressions are shown in figure 4 together with the available experimental results of Fekete ${ }^{(12)}$, and Wilson and Goldstein ${ }^{(13)}$. In this regard the review by Launder and Rodi(14) suggest that the data of Fekete is possibly more representative of two dimensional flow than that of Wilson and Goldstein. It is in fairly good agreement with the theoretical expression for $n=6$ (the Reynolds number is in the range 4 to $13 \times 10^{3}$ ).

\section{(b) Iogarithmic Spiral}

For a logarithmic spiral $\mathrm{R} / \mathrm{s}=$ constant and equation (22) has a self similar solution

$$
\begin{aligned}
b / s & =\frac{k\left[1+\frac{n}{k} b / R\right]}{\left[1-\frac{\frac{10}{12}-\log 2}{k} b / R\right]} \\
& \approx .07 \frac{[1+8 b / R]}{[1-b / R]} \quad(n=7) \\
& \approx \frac{.07[1+6.8 b / R]}{[1-b / R]} \quad(n=6)
\end{aligned}
$$

A comparison of these curves with the experimental results of Guitton and Newman ${ }^{(14)}$ and Kamemoto ${ }^{(15)}$ is made in fig. 5. Here Guitton and Newman's results are considered to be most representative of two dimensional flow. Equation (24) reflects the much greater increase of $b / s$ with $b / R$ for the logarithmic spiral, compared to the circular cylinder equation (23a), and this trend is also seen in the experimental data.

\section{Conclusions}

From the foregoing analysis and comparison with experimental results it is seen that the wall jet is influenced by three effects: 
(a) The turbulent diffusion in the wall jet giving rise to a linear spreading rate $d b / d s=$ $K \approx .07$.

(b) A coupling between the eddy viscosity and the curvature of the wall arising from the term $\frac{\partial}{\partial y}(\epsilon u / R)$ in the stress gradient and yielding a contribution of $\frac{d b}{d \bullet}=K \frac{n}{k} b / R$. (typically this contribution is $O\left(\frac{1}{2} b / R\right)$ for Reynolds numbers typical of laboratory experiments), and

(c) A curvature-induced streamwise pressure gradient giving rise to a contribution of $O(b / R)^{2} \frac{d R}{d \theta}$. When $\frac{d R}{d S}<O$ this effect causes the jet momentum $\left(b u_{m}^{2}\right)$ to increase in the streamwise direction and help confine the jet to a thin layer as it proceeds along the surface. This is an inertial term, largely independent of Reynolds number, and is an essential feature of Coanda turning of the flow.

These influences are additive and give an approximate relationship between the spreading rate $\frac{d b}{d t}$, the half width of the jet and the surface radius of curvature $R$, of the form

$$
\frac{d b}{d s}=K_{0}+K_{1} \frac{b}{R}+K_{2}\left(\frac{b}{R}\right)^{2} \frac{d R}{d s}
$$

which can be integrated to give $b(s)$ when the wall shape $R(s)$ is known.

The use of this result for the circular cylinder $(R=$ const $)$ and for logarithmic spiral surfaces $(\mathrm{b} / \mathrm{R}=$ const) shows good agreement with the previcus experimental result3 of several authors. This provides some confidence that a simple algebraic eddy viscosity model, used in conjunction with the apprcpriate expression for the shear stress (including curvature terms), is sufficient to describe the primary features of the wall jet. 


\section{REPERENCPS}

1. Young, T., "Outline of Experiments and Inquiries Regarding Sound and Light", Lecture to the Royal Society, January 16, 1800 (see Journal Royal Aeronautical Society Vol. 61 1957, p 10157).

2. Wood, N. and Nielsen, J., "Circulation Control Airfoils, Past, Present and Future", AIAA paper 85-0204, January 14-17, 1985.

3. Englar, R. J., "Experimental Investigation of the High Velocity Coanda Wall Jet Applied to Bluff Trailing Edge Circulation Control Airfoils”, NSRDC TN AL-308.

4. Launder, B. A. and Rodi, W., "The Turbulent Wall Jet", Progress in Aerospace Sciences, Vol. 19, pp. 81-128, Pergaman Press Ltd 1981.

5. Tailland, A., and Mathieu, J., "Jet Paretial", Journal de Mecanique $\underline{6}, 103,1967$.

6. Tailland, A., "Contribution à l' étude d'un jet plan dirige tangentiellement à une paroi plane", Thes̀e de Docteur es Sciences, Univ. Claude Bernard, Lyon 1970.

7. Forthmann, E., “Uber Turbulent Strahlausbreitung”, Ing Arch., $\underline{5}, 42,1934$.

8. Sigalla, A., "Measurements of Skin Friction in a Plane Turbulent Wall Jet", J. Royal Aeronautical Society $\underline{62}$ 873, 1958.

9. Bradshaw, P., and Gee, M. T., "Turbulent Wall Jets with and without an External Stream", Aero. Research Council, R \& M 3252, 1960.

10. Patel, R. P., "Self-Perserving, Two-Dimensional Turbulent Jets and Wall Jets in a Moving Stream", M. Eng. Thesis, Dept. of Mechanical Engineering, McGill University, Montreal, 1962.

11. Giles, J. A., Hays, A. P., and Sawyer, R. A., "Turbulent Wall Jets on Logarithmic Spirai Surfaces", Aero Quart., 17 201, 1966.

12. Felrete, G. I., "Coanda Flow in a Two-Dimensional Wall Jet on the Outside of a Circular Cylinder" Mech. Engr. Dept. Rep. No. 63-11, McGill University 1963

13. Wilson D. J., and Goldstein, R. J., "Turbulent Wall Jets With Cylindrical Streamwise Surface Curvature”, J. Fluids Eng., $\underline{98}$ 550, 1976.

14. Guitton, D. E. and Newman, B. G., "Self Preserving Turbulent Wall Jets over Convex Surfaces", J. Fluid Mech. $\underline{81}$ 155, 1977.

15. Kamemoto, K., "Investigation of Turbulent Wall Jets over Logarithmic Spiral Surfaces", Bulletin JSME 173331974. 


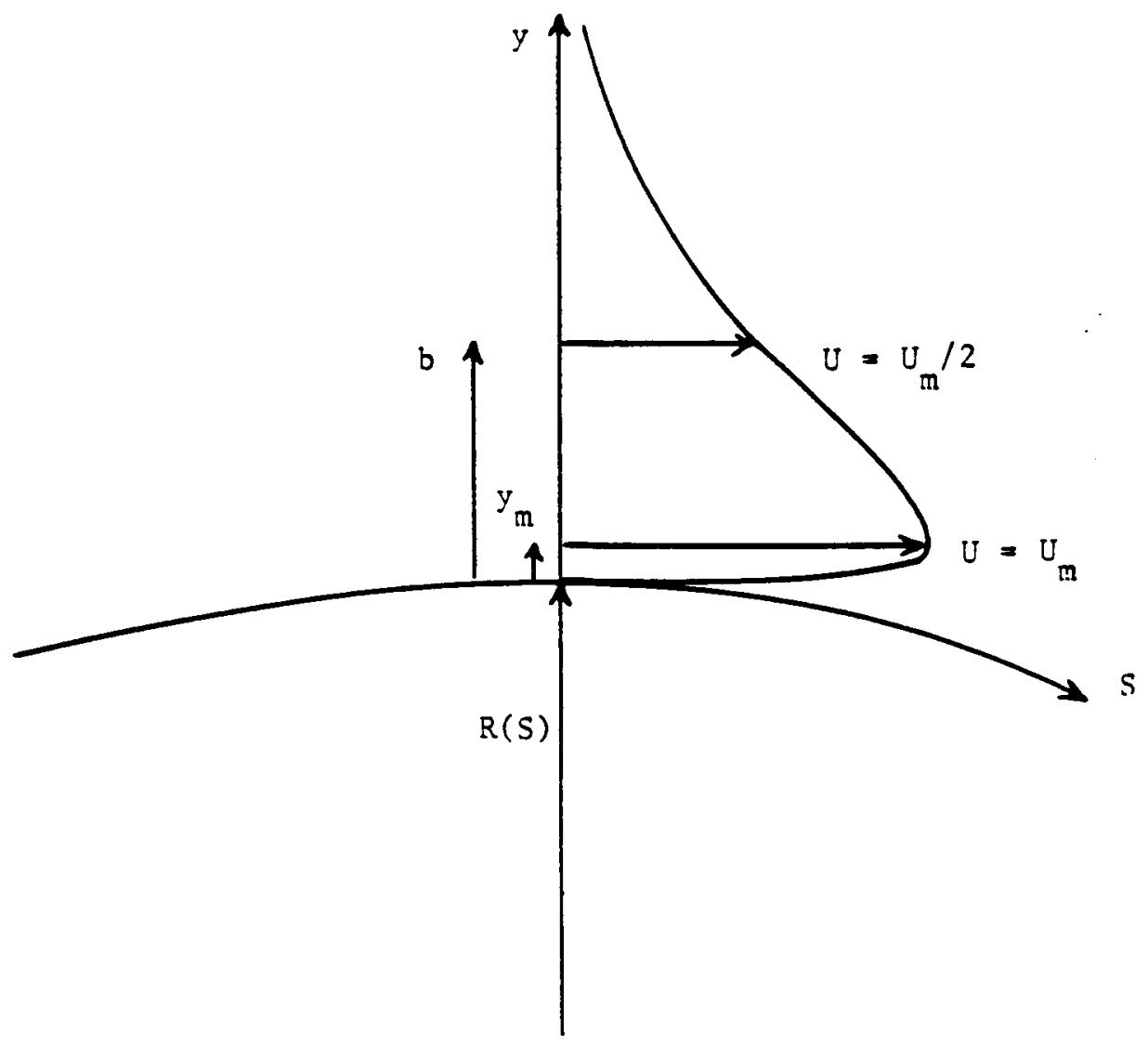

Figure 1. Geometry of two dimensional wall-jet. 


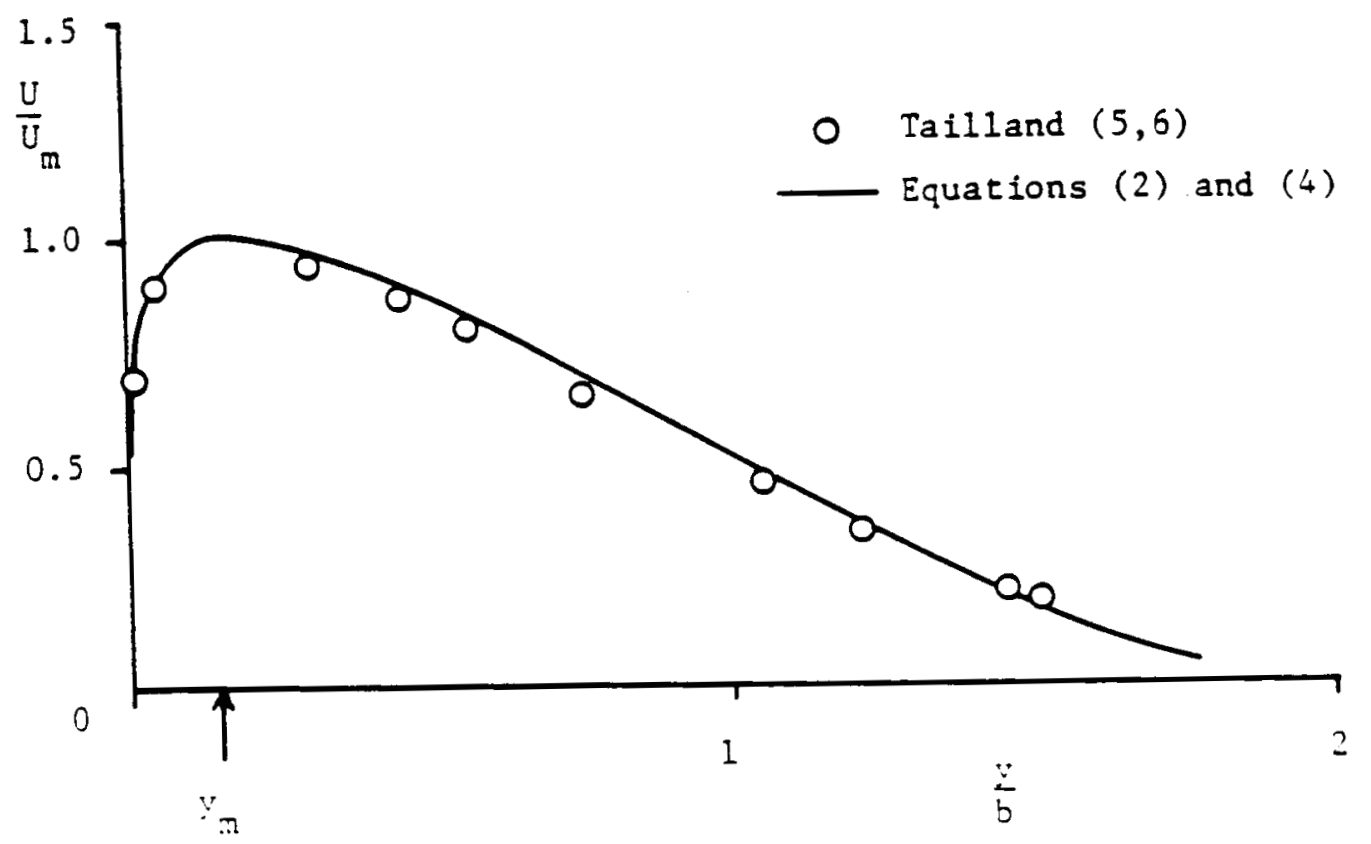

Figure 2. Comparison of assumed velocity profile with experimental data. 


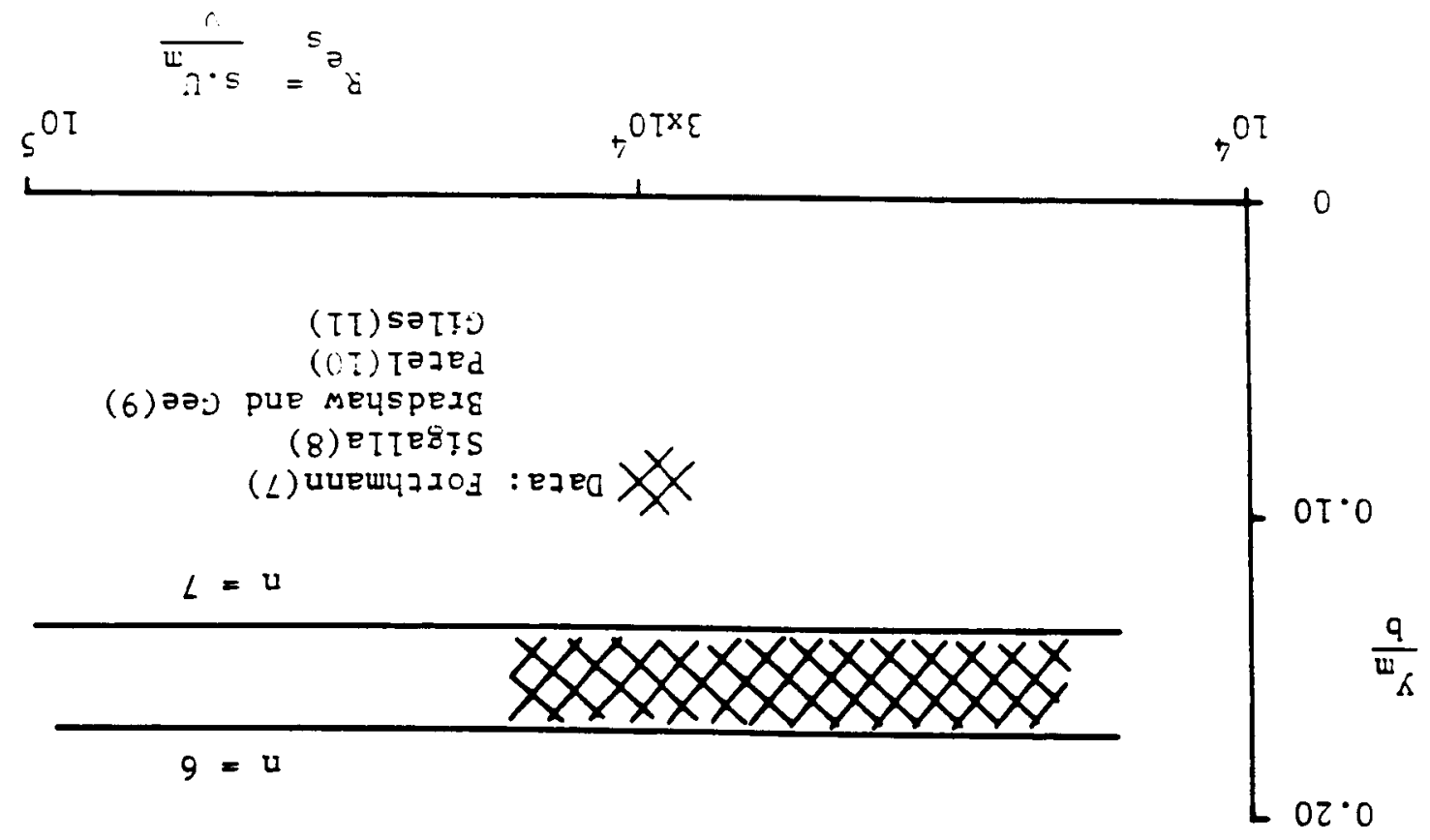




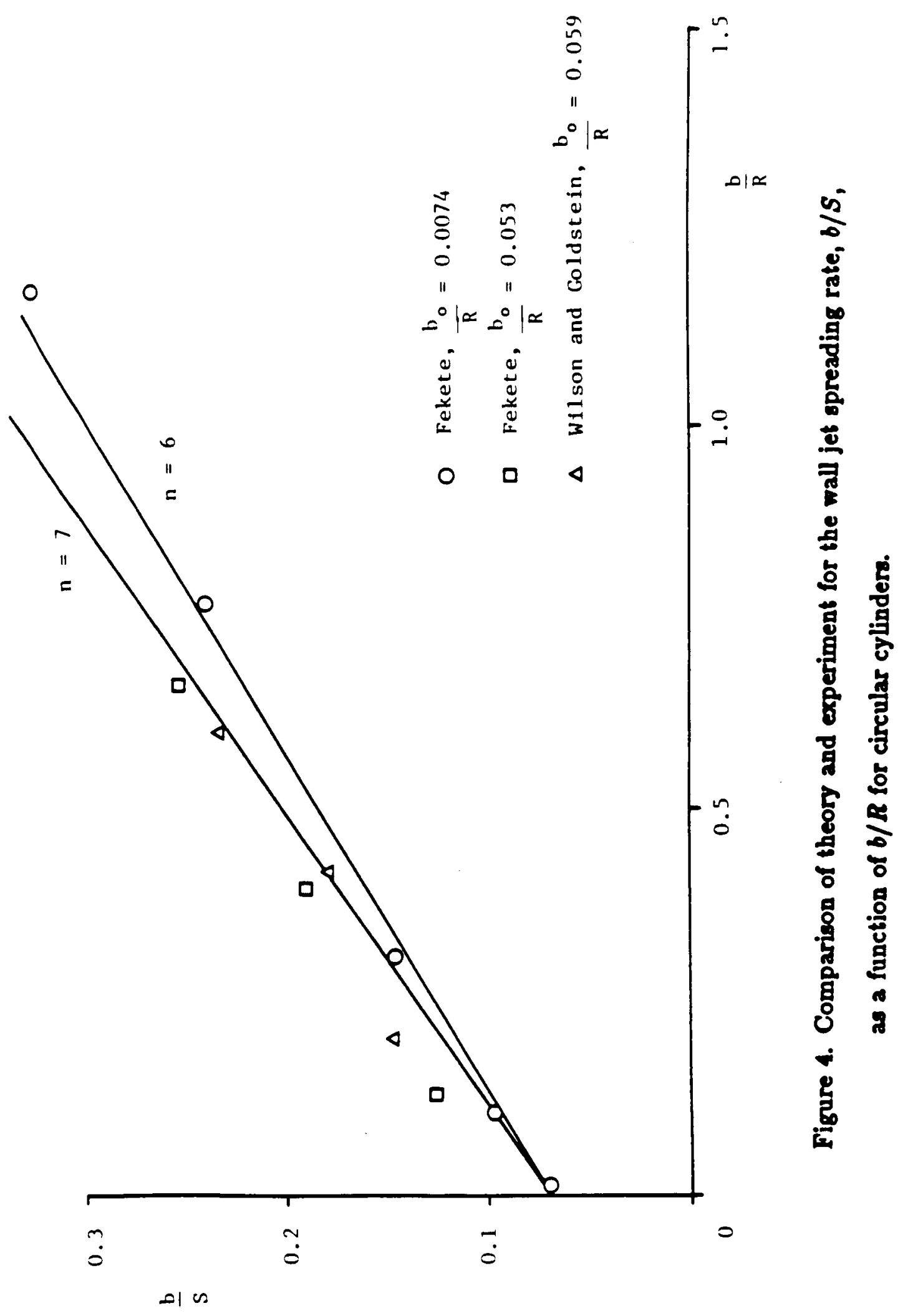



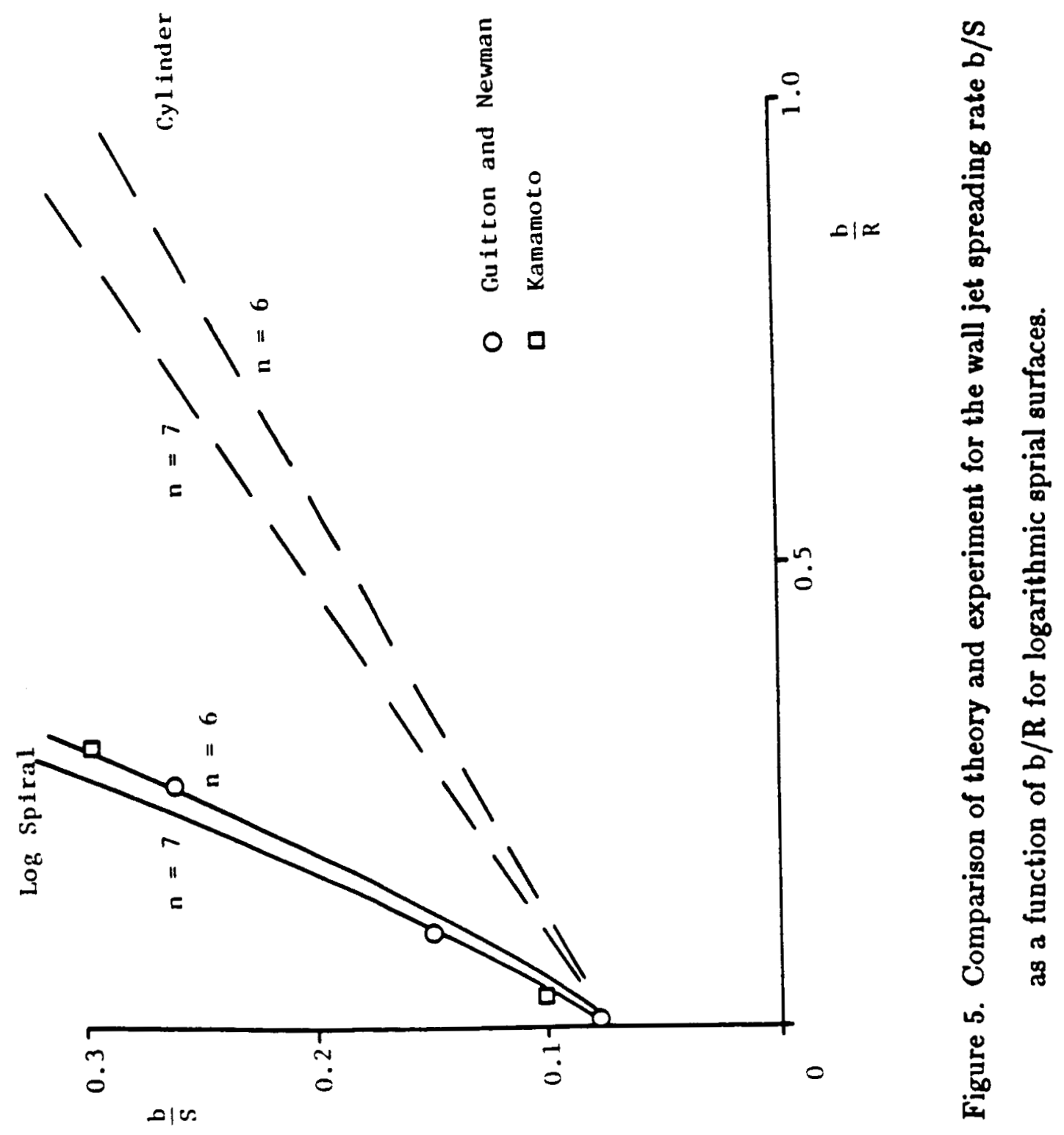